\title{
The Application of the Improved Chaos Algorithm in the Sports Performance Prediction
}

\author{
Suqiong Feng \\ Sichuan Agricultural University. China \\ fengtingting0828@163.com
}

Keywords: Physical Training, Training plan, Chaos algorithm.

\begin{abstract}
With the continuous progress of the science, the physical training is also gradually scientific. As a kind of important sports phenomenon and rule, the statistics and the quantitative analysis method in the sports fieldwill be paid more and more attention by people. During the training process, using the scientific methods to predict the sports performance can find out the game rules of the players and make the training plans. In this paper, we use the chaos prediction method to predict the sports performance. In order to improve the performance of the algorithm, we improve the traditional chaos prediction algorithm and propose the improved chaos algorithm. According to the examples, we analyse the accuracy of the prediction.
\end{abstract}

\section{Introduction}

It is the current developing trend to combine the sports with the prediction. The scholars studied the prediction of the sports industry (Tongren Xia, Maosen Jiang (2015)) and the prediction of sports talents (Daojing Chen, Xiaoman Zhao (2006)). The improvement of the sports performance cannot be separated from scientific training. The prediction of the sports performance can help the coach analyze the rules of the game. Then, that can formulate the scientific and reasonable training plan.In the modern sports development, the professional trainers will find rules from training data and help the athletes for the sports training. Under this background, it is very necessary to predict the sports performance.

The Chaos theory is an important part of the nonlinear theory (Fr D ricAndr (2016)). The sensitive characteristics of the initial value for the chaotic system makes the changes of the system input can be reflected quickly in the output. The feature indicates that the chaotic model is closer to the reality of the world (Konrad Kolesko, RafaLata a (2015)).

In the long development course, the Chaos theory unceasingly consummates. The research direction has included the chaotic dynamics (d'Aquino et al. M. (2016)), the chaos prediction (Heydari et al. Gholamali (2016)), the chaos control (Chattopadhyay et al. Joydev (2015)) etc. with the urgent demand of the social practice to the prediction theory and the limitations of the traditional forecasting models, the chaos prediction has become a focus of the chaos theory study (Wu et al. Jun (2009)).Therefore, in this paper, we choose the chaos prediction theory to study. It has the great theoretical value and the practical significance.

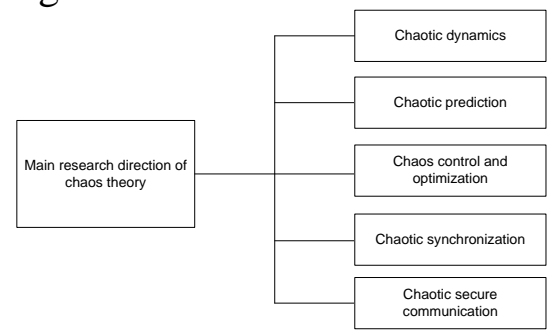

Figure 1. The main research direction of chaos theory

In the long development course, the Chaos theory unceasingly consummates. The research direction has included the chaotic dynamics, the chaos prediction, the chaos control etc.

In this paper, we study the prediction of the sports performance. By using the thought of chaos prediction, we forecast the sports performance. In order to achieve a good prediction effect, we put 
forward the improved chaos algorithm. Then, we use the algorithm to study and predict the athletes' sports performance. Finally, the experiment shows that the algorithm which applies to the sports performance prediction has achieved the good results.

\section{The chaos algorithm}

Li Yorke definition gives the first mathematical definition of the chaos. It is the first time that the scholar gives the chaos the strict scientific significance. It is the chaos mathematical definition which has the great influence. It is defined from the interval mapping. For the continuous self-mapping on the interval, if it satisfies the following conditions, it can have the chaos phenomenon.

(1) For the periodic point $f$, the periodic has no the upper bound. $f$ has the periodic points with arbitrary positive integer period. That is, for any natural number ${ }^{n}$, there is $x \in I$ making $f_{n}(x)=x$.

(2) For the closed interval $I$, there exists $S$ satisfying,

When $\forall x, y \in S, x \neq y$

$$
\lim _{n \rightarrow \infty} \sup \left|f^{n}(x)-f^{n}(y)\right|>0
$$

When $\forall x, y \in S$

$$
\liminf _{n \rightarrow \infty}\left|f^{n}(x)-f^{n}(y)\right|=0
$$

When $\forall x \in S$ and the any periodic point of $f$ is $y$,

$$
\limsup _{n \rightarrow \infty}\left|f^{n}(x)-f^{n}(y)\right|>0
$$

$\sup \left|f^{n}(x)-f^{n}(y)\right|$ is the upper bound of the distance between $f^{n}(x)$ and $f^{n}(y) . \inf \left|f^{n}(x)-f^{n}(y)\right|$ is the lower bound of the distance between $f^{n}(x)$ and $f^{n}(y)$.

Li-York theorem: we assume that $f(x)$ is the mappingin $[a, b]$. If $f(x)$ has three periodic points, for any positive integer $n, f(x)$ has $n$ periodic points.

Taken theorem: $M$ is $d$ dimensional space, $\varphi: M \rightarrow M, \varphi$ is a smooth diffeomorphism. $y: M \rightarrow R,{ }^{y}$ has two order continuous derivative. $\phi(\varphi, y): M \rightarrow R^{2 d+1}$, where,

$\phi(\varphi, y)=\left(y(x), y(\varphi(x)), y\left(\varphi^{2}(x)\right), \cdots, y\left(\varphi^{2 d}(x)\right)\right)$

Therefore, $\phi(\varphi, y)$ is a embed from $M$ to $R^{2 d+1}$.

There are many ways to choose the delay time and embedding dimension. According to the embedding theorem, if $m \geq 2 d+1$, we can reveal the chaotic attractor. There are many ways to choose the embedding methods. We introduce the Cao method and the C-C method.

The Cao method was proposed by Cao Liangyue in 1997. Firstly, we define,

$$
a(i, m)=\frac{\left\|Y_{i}(m+1)-Y_{n(i, m)}(m+1)\right\|}{\left\|Y_{i}(m)-Y_{n(i, m)}(m)\right\|}
$$

Where, $Y_{i}(m+1)$ is the $i$ vector in $(m+1)$ dimension reconstructed phase space. In $m$ dimension reconstructed phase space, $n(i, m)$ makes $Y_{n(i, m)}(m)$ as the nearest neighbor integer of $Y_{i}(m) . n(i, m)$ is determined by $i$ and $m$. $\|\cdot\|$ isEuclid records. It can also be calculated and achieved by the maximum norm. That is,

$$
\left\|X_{p}(p)-X_{i}(p)\right\|=\max _{0 \leq j \leq p-1}\left|x_{k+j \tau}-x_{l+j \tau}\right|
$$

We define that all the average value of $a(i, m)$ is,

$$
E(m)=\frac{1}{N-m \tau} \sum_{i=1}^{N-m \tau} a(i, m)
$$

Then, we define the change from $m$ to $m+1$,

$$
E 1(m)=E(m+1) / E(m)
$$

In Cao method, at the same time, we use the parameter to distinguish the new good and random signal of the deterministic chaos. That is,

$$
E^{*}(m)=\frac{1}{N-m \tau} \sum_{i=1}^{N-m \tau}\left|x_{i+m \tau}-x_{n(i, m)+m \tau}\right|
$$




$$
E 1(m)=E^{*}(m+1) / E^{*}(m)
$$

For each time series of the $\mathrm{C}-\mathrm{C}$ method, $\mathrm{C}-\mathrm{C}$ method adopts the following to calculate.

$$
\begin{aligned}
& \bar{S}(\tau)=\frac{1}{16} \sum_{m=2}^{7} \sum_{j=1}^{4} S\left(m, r_{j}, \tau\right) \\
& \Delta \overline{S_{1}}(\tau)=\frac{1}{4} \sum_{m=2}^{7} \Delta S(m, \tau) \\
& S_{c o r}(\tau)=\Delta \overline{S_{1}}(\tau)+|\bar{S}(\tau)|
\end{aligned}
$$

$S\left(m, r_{j}, \tau\right)=C\left(m, r_{j}, \tau\right)-C^{m}\left(1, r_{j}, \tau\right)$ reflects the system's self- correlation characteristic. $r$ is the size of the neighborhood radius.

$C(m, r, \tau)=\frac{1}{M^{2}} \sum_{1 \leq j \leq k \leq M} \theta\left(r-\left\|X_{j}-X_{k}\right\|\right)$ is the correlation integral of the system. $\theta(\cdot)$ is the Heaviside function.

$$
\theta(x)=\left\{\begin{array}{l}
0, x<0 \\
1, x \geq 0
\end{array}\right.
$$

For the estimation of the correlation dimension, we adopt G-P algorithm [22]. When $r \rightarrow 0, N \rightarrow \infty$, the associated integral function is approximated to the following function.

$\log _{2} C(m, r, \tau) \rightarrow v \log _{2} r$

When $m$ continues to increase, the limit value of $v$ is the correlation dimension of the system.

We select the first zero point of $\bar{S}(t)$ or the first minimum point which goes through $\Delta \bar{S}(t)$ at the first time as the most appropriate time delay $\tau$. The minimum point of $S_{c o r}(r)$ is the optimal time delay window.

\section{Improved chaos prediction method}

During the prediction process of the chaotic time series, the selection of the embedding dimension and delay time is the basis of chaotic time series prediction. At present, there are a lot of computation methods (Li et al. Shuang (2011)). The same chaotic time series can be embedded in different (or multiple) phase space. The predicted results are also different. In order to improve the reliability and practicability of the chaotic time series prediction, we propose a newmulti-embedding dimension method. Near the obtained embedding dimension, the method takes several different dimensional numerical and calculate the load forecasting results under different embedding dimension. Finally, we weight and average the obtained results and they are the final prediction value.

For the known time series $\{x, i=1,2, \cdots, N\}$, firstly, according to the certain embedding dimension and the delay time calculation method, we calculate the embedding dimension $m$ and the delay time $\tau$. Then, we reconstruct the phase space and establishthe first order linear prediction model. In general, the different embedding dimension calculation method or the difference of the artificial subjective factor can make the different value of the embedding dimension. The predicted resultsare also different in different embedding dimensions. We assume that there are $k$ different embedding dimensions. In each embedding dimension, the prediction result of $x(n+1)$ is $x_{1}(n+1), x_{2}(n+1) \cdots, x_{n}(n+1)$.

The prediction error is ,

$$
\Delta e_{i}=x_{i}(n+1)-x(n+1), i=1,2, \cdots, k
$$

Where, $x(n+1)$ is the actual value. $x_{i}(n+1)$ is the prediction value of the $i$ embedding dimension. We assume that the prediction average value in $k$ embedding dimensionis $x_{p}$. That is,

$$
x_{p}=\left(x_{1}(n+1), x_{2}(n+1), \cdots x_{k}(n+1)\right) / k
$$

The error of each prediction is,

$$
r_{i}=x_{i}(n+1)-x_{p}
$$

We assume that the probability that we obtain $r_{i}$ is, 


$$
p\left(r_{i}\right)=\frac{1}{\Delta e_{i} \sqrt{2 \pi}} e^{-\frac{r_{i}^{2}}{2 \Delta e_{i}^{2}} d r_{i}}
$$

Because the prediction results are independent in different embedding dimensions, the occurrence probability for all $k$ is,

$$
p=\prod_{i=1}^{k} p\left(r_{i}\right)
$$

If $p$ is bigger, it shows that the prediction results are reliable. That is, when $\min \sum_{i=1}^{k} r_{i}^{2}$, the prediction results are the most reliable. Therefore, according to the least square method, the final prediction result in different embedding dimension is,

$$
x(n+1)=\frac{\sum_{i=1}^{k} p\left(r_{i}\right) x_{i}(n+1)}{\sum_{i=1}^{k} p\left(r_{i}\right)}
$$

Where, $x_{i}(n+1)$ is the prediction value of the $i$ embedding dimension at $n+1$ time. $p\left(r_{i}\right)$ is the probability of the prediction error for the $i$ embedding dimension.

In the phase space, any two adjacent tracksdiverges with the trend that largest lyapunovexponents $\lambda_{1}$. That is, in normal circumstances, if the initial two state displacement $d(0)$ is not entirely along the vertical $\lambda_{1}$, we can easily get,

$$
d(t)=d(0) e^{\lambda_{1} t} \rightarrow \operatorname{Ind}(y)=\operatorname{ind}(0)+\lambda_{1} t
$$

We assume that there is a time series. We use it to reconstruct the phase space. We select arbitrarily two adjacent initial positions. In general, the two points are the initial point and the nearest neighbor. Then, we calculate the initial distance $d(0)$ of the two points. Next, we select appropriately the step size $\Delta t$ and the time length $t=k \Delta t$. We calculate the distance $d(k \Delta t)$ that elapsing the time $t$. Then, we can get,

$$
\lambda_{1}=\frac{1}{k \Delta t} \operatorname{In} \frac{d(k \Delta t)}{d(0)}
$$

We select the different initial distance $d_{j}(0)$ to calculate $d_{j}(k \Delta t)$ and calculate the average value.

$$
\lambda_{1}=\frac{1}{k \Delta t} \operatorname{In} \frac{d_{j}(k \Delta t)}{d_{j}(0)}
$$

\section{Experiment}

We take the sports school 1500 meter race as the example. We apply the improved chaos method to predict the 1500 meter long-distance race performance. We select the performance of 4 athletes to predict. Among them, the first 20 data are as the training set and the last 10 data are as the prediction set. The training set data are shown in the following table. 
Table 1. Data of the training set

\begin{tabular}{ccccc}
\hline No & Play 1 & Play 2 & Play 3 & Play 4 \\
\hline 1 & 275 & 243 & 257 & 248 \\
2 & 277 & 248 & 246 & 241 \\
3 & 265 & 245 & 253 & 244 \\
4 & 266 & 243 & 258 & 237 \\
5 & 268 & 238 & 253 & 235 \\
6 & 273 & 235 & 254 & 238 \\
7 & 272 & 233 & 246 & 243 \\
8 & 270 & 230 & 244 & 246 \\
9 & 265 & 236 & 241 & 237 \\
10 & 267 & 231 & 242 & 240 \\
11 & 262 & 230 & 238 & 241 \\
12 & 268 & 226 & 235 & 249 \\
13 & 264 & 234 & 241 & 247 \\
14 & 265 & 237 & 232 & 248 \\
15 & 266 & 243 & 240 & 251 \\
16 & 259 & 238 & 245 & 254 \\
17 & 261 & 232 & 244 & 247 \\
18 & 263 & 234 & 251 & 244 \\
19 & 258 & 236 & 246 & 239 \\
20 & 260 & 233 & 243 & \\
\hline
\end{tabular}

We put the data in a figure.

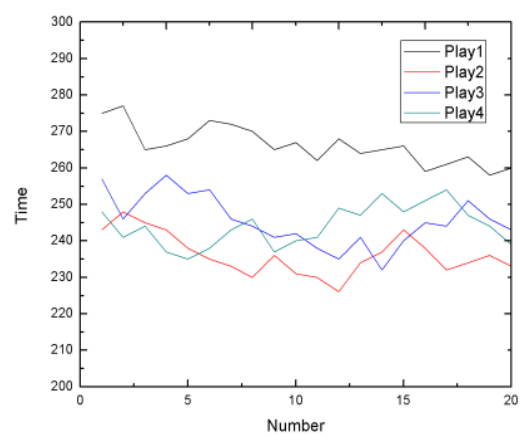

Figure 2.Data of the training set

Then, we predict the performances. The prediction value and the actual value are as follows.

Table 2.The actual values and the predicted values

\begin{tabular}{ccccccccc}
\hline No & \multicolumn{2}{c}{ Play1 } & \multicolumn{2}{c}{ Play 2 } & \multicolumn{2}{c}{ Play 3 } & \multicolumn{2}{c}{ Play 4 } \\
\hline & Actual & Predicted & Actual & Predicted & Actual & Predicted & Actual & Predicted \\
& results & results & results & results & results & results & results & results \\
21 & 257 & 258 & 238 & 240 & 248 & 249 & 241 & 244 \\
22 & 264 & 266 & 237 & 239 & 246 & 247 & 248 & 250 \\
23 & 261 & 264 & 235 & 237 & 254 & 254 & 247 & 251 \\
24 & 253 & 256 & 236 & 236 & 246 & 248 & 253 & 252 \\
25 & 255 & 255 & 231 & 233 & 247 & 244 & 256 & 254 \\
26 & 260 & 261 & 230 & 231 & 245 & 246 & 249 & 252 \\
27 & 265 & 263 & 235 & 233 & 244 & 245 & 248 & 247 \\
28 & 257 & 261 & 233 & 234 & 249 & 247 & 242 & 246 \\
29 & 259 & 260 & 231 & 235 & 245 & 240 & 241 & 242 \\
30 & 261 & 262 & 235 & 234 & 243 & 241 & 239 & 240 \\
\hline
\end{tabular}


From the above table, we can see that the actual values and the predicted values of the four athletes are very similar. The errors are very small. It shows that the improved chaos algorithm has achieved the good results for the sports performance. The experimental results demonstrate furtherly the effectiveness of the improved algorithm.

\section{Conclusion}

The development of the sports cannot have the science. Training the sports by the scientific way can find out the rule, improve the training performance of the athletes and promote the development of the sports. Chaos prediction is a mature forecasting method. In this paper, we use the chaos prediction method to study the prediction of the sports performance. At the same time, we put forward the improved chaos prediction method to make the result more accurate. Firstly, this paper introduces the basic theory of the chaos prediction. Then, this paper puts forward the improved chaos algorithm.In the experimental part, we use the improved chaos algorithm to predict the performance of the athletesand achieve the good experimental results.

\section{References}

[1]. Frédéric André,2016, A polynomial chaos approach to narrow band modeling of radiative heat transfer in non-uniform gaseous media, Journal of Quantitative Spectroscopy and Radiative Transfer, 175: 17-29, DOI: 10.1016/j.jqsrt.2016.01.037.

[2]. GholamaliHeydari, MohammadAli Vali, Ali Akbar Gharaveisi, 2016, Chaotic time series prediction via artificial neural square fuzzy inference system, Expert Systems with Applications, 55: 461-468, DOI: 10.1016/j.eswa.2016.02.031.

[3]. Jiang Tongren, Xia Maosen, 2015, Development of Chinese sports industry under new norm, Journal of Wuhan Institute of Physical Education, 5:47-55, DOI: 10.15930/j.cnki.wtxb.2015.05.008.

[4]. Joydev Chattopadhyay, Nikhil Pal, Sudip Samanta, Ezio Venturino, Q.J.A. Khan,2015, Chaos control via feeding switching in an omnivory system, Biosystems, 138: 18-24, DOI: 10.1016/j.biosystems.2015.10.006.

[5]. Jun Wu, Jian Lu, Jiaquan Wang, 2009, Application of chaos and fractal models to water quality time series prediction, Environmental Modelling \& Software, 24: 632-636, DOI: 10.1016/j.envsoft.2008.10.004.

[6]. Konrad Kolesko, RafałLatała, 2015, Moment estimates for chaoses generated by symmetric random variables with logarithmically convex tails, Statistics \& Probability Letters, 107: 210-214,DOI: 10.1016/j.spl.2015.08.019.

[7]. M. d'Aquino, A. Quercia, C. Serpico, G. Bertotti, I.D. Mayergoyz, S. Perna, P. Ansalone, 2016 Chaotic dynamics and basin erosion in nanomagnets subject to time-harmonic magnetic fields, Physica B: Condensed Matter, 486: 121-125, DOI: 10.1016/j.physb.2015.09.032.

[8]. Shuang Li, Qian Li, Jiaorui Li, Jinqian Feng, 2011, Chaos prediction and control of Goodwin's nonlinear accelerator model, Nonlinear Analysis: Real World Applications, 12:1950-1960, DOI: 10.1016/j.nonrwa.2010.12.011.

[9]. Zhao Daojing, Chen Xiaoman, 2006, Prediction of needs for sports talents and development countermeasures, Journal of Wuhan Institute of Physical Education, 12:31-35, DOI: 10.15930/j.cnki.wtxb.2006.12.007. 